\title{
Consideraciones en las estrategias didácticas de las giras de campo en un modelo de educación a distancia
}

\author{
Rosita Ulate SÁnchez. 2013
}

Recibido: 18 julio 2013

RESUMEN

Uno de los resultados de la primera fase cualitativa de la investigación Mixta, Valoración Participativa de las Características Técnico Pedagógicas y Usos Académicos de un Material Multimedial para las Giras de Campo, se vinculó a la recuperación de la importancia de las actividades vivenciales en el proceso de enseñanza y aprendizaje de un modelo de educación a distancia. Ellas promueven el desarrollo de habilidades y competencias a través de la práctica de los conocimientos teóricos adquiridos. De allí que las giras de campo, en carreras en el área de los recursos naturales, permita desarrollar en los estudiantes las competencias requeridas como futuros profesionales. La ponencia Consideraciones en las Estrategias Didácticas de las Giras de Campo en un Modelo de Educación a Distancia evidencia la necesidad de planificar las giras de campo bajo un modelo de estrategia didáctica, asegurando la calidad y el cumplimiento de los objetivos académicos y de seguridad de una actividad vivencial dentro de un modelo a distancia.

Palabras clave: Estrategias didácticas, Giras de Campo, Actividades Vivenciales, Recursos Naturales.

\begin{abstract}
One of the results of the first qualitative phase of the joint research Participatory Rating of Technical and Pedagogical Uses of Multimedia Material for Field Visits, Technical Specifications are linked to the recovery of the importance of experiential activities in the teaching-learning process of a distance education model. They promote the development of skills and competencies through practice of the acquired theoretical knowledge. Hence, the field trips, in the area of natural resources, will enable students to develop the skills required as future professionals. The paper Considerations in the Teaching Strategies of Field Visits in a Model of Distance Education highlights the need to plan field trips under a teaching strategy model, ensuring quality and meeting of academic and safety goals of an experiential activity within a distance model.
\end{abstract}

Key words: Teaching Strategies, Field Visits, Experiential Activities, Natural Resources.
Aceptado: 27 agosto 2013

\section{Introducción}

La modalidad de educación superior a distancia para la Universidad Estatal a Distancia (UNED) ha sido entendida como una enseñanza semi-presencial de la formación profesional y de la educación abierta, centrada en el estudiante (Guadamuz, 2008).

En general, la formación profesional universitaria de las ciencias naturales en la Escuela de Ciencias Exactas y Naturales (ECEC) ha estado sustentada en un currículo con diseños de cursos que mantienen un porcentaje importante de participación y experiencia de los estudiantes en laboratorios, giras y trabajos de campo, y exposición a escenarios y situaciones reales (UNED, 2008b).

En la Carrera de Manejo de Recursos Naturales, en estudio, al menos el 29\% de los cursos del programa de estudios completo hasta el 2012 fue mediado a través de giras de campo, implementando el conocimiento vivencial de los estudiantes (UNED, 2008a). Por ello, en dicha carrera se persigue el desarrollo de características en el perfil profesional, que generalmente es promovido por medio de actividades de aprendizaje con la construcción del conocimiento en las giras de campo, las cuales se establecen como iniciativas pedagógicas, de llevar la teoría a la práctica y que, como lo establece la Alianza Cambio Andino (2008), se llevan a cabo "con el propósito de promover el intercambio horizontal de conocimientos entre distintos actores en un área específica y motivar el interés para la réplica de experiencias similares (...) conocimiento vivencial a través de visitas a experiencias, sobre las que interesa entender su desarrollo, resultados y principales lecciones aprendidas" (p. 1). 


\section{Metodología}

Los hallazgos de la presente ponencia corresponden a uno de los componentes de la primera de cuatro fases que completaron la disertación aplicada Valoración Participativa de las Características Técnico Pedagógicas y Usos Académicos de un Material Multimedial para las Giras de Campo. El estudio, presentada como una disertación aplicada se realizó a partir del paradigma constructivista (Driscoll, 2005) y de algunos elementos del paradigma sociocrítico (Arnal, 1996), se propuso una investigación con un diseño mixto complejo con niveles múltiples (Creswell, 2003; Creswell \& Plano, 2007; Hernández, Fernández \& Baptista, 2006; Tashakkori \& Teddlie, 1998) ya que se recolectaron, analizaron y vincularon datos cualitativos y cuantitativos mediante cuatro fases secuenciales.

La primera fase comprendió un estudio integral, predominantemente cualitativo, del programa de estudio de MARENA, en el cual se determinaron las generalidades de la carrera y de las giras de campo, mediante entrevistas a profundidad en una sesión de grupo heterogénea.

La muestra de los participantes en la investigación fue seleccionada, dirigida y voluntaria por estratos. Los participantes del estudio son docentes y estudiantes de la Escuela de Ciencias Exactas y Naturales (ECEN) de la institución de estudio; todos funcionarios relacionados con diseño, implementación, evaluación, dirección, asesoría en el diseño y producción de materiales multimedia de las giras de campo de los cursos de MARENA.

Los resultados obtenidos que se presentan en esta ponencia responden a uno de los pasos que se desarrolló en la investigación realizada bajo la pregunta de investigación: ¿Cuáles características técnico-pedagógicas y usos académicos se deben contemplar para producir un material multimedial, como parte del proceso formativo de autoaprendizaje, en las giras de campo de MARENA?

En la primera fase de análisis se contó con un total de 17 personas de la institución de estudio, de las cuales nueve correspondieron al nivel I (un encargado de programa y ocho encargados de cátedra); cuatro al nivel II (cuatro profesores) y cuatro al nivel III (cuatro asesores y expertos). Para la sesión de grupo realizada se utilizó una guía de sesión de grupo, (preparada por la investigadora y validada por expertos de la universidad de estudio). Este instrumento tubo como propósito promover la discusión y conocer la opinión de los informantes con respecto a tópicos como la información general de la carrera, de las cátedras y de los cursos con giras de campo. Adicionalmente, se dio especial interés a las experiencias de los entrevistados en el tema de las giras de campo de MARENA y hacia el proyecto de investigación en desarrollo; clasificación de las giras de campo y objetivos.

La información obtenida en la primera fase fue predominantemente de tipo cualitativa, fue agrupada en categorías y fueron procesadas por medio del software Word de Microsoft Windows $2007^{\circledR}$.

\section{Revisión de Literatura}

Las primeras concepciones de las giras de campo se basaron en teorías de aprendizaje con influencia del conductismo, en las que imperaba un enfoque epistemológico de la filosofía empirista y positivista de la objetividad. Según esta filosofía, la adquisición del conocimiento se explica como producto de la experiencia, concentrándose en la descripción de los hechos observables (ajenos a los intereses del investigador) y por medio de procesos generalizados y de inducción (Driscoll, 2005).

Por otro lado, las concepciones posteriores de las giras de campo siguieron los aportes de las teorías de aprendizaje del desarrollo cognitivo (cognición situada) y del constructivismo, desde una perspectiva más cercana a la expuesta por Bruner y Vigostsky (Driscoll, 2005). En dicho enfoque, el objeto de estudio y el investigador están interrelacionados y el descubrimiento del conocimiento ocurre en el proceso de investigación, a través del cual, epistemológicamente, hay una discusión crítica y una construcción subjetiva del conocimiento (Guba, 1990). Desde la cognición situada, tal construcción subjetiva es llamada por Kirshner y Whitson (1997) como 
aprender haciendo, a través de aquello que los expertos en la materia hacen o enseñan a realizar. En esta segunda visión de la gira de campo, el estudiante es un sujeto activo en la construcción de su aprendizaje a partir de la interacción con el medio y el contexto social, y el aprendizaje es mediado por un facilitador en un contexto de una experiencia vivencial del estudiante.

Las giras de campo son actividades de aprendizaje que pueden llegar a responder al planteamiento de Tovar (2008), quien indica la necesidad de buscar iniciativas desde la dinámica de las ciencias naturales para proponer el aprendizaje y la enseñanza con dimensiones conceptuales, metodológicas, actitudinales y comunicativas ante contextos políticos, ambientales, económicos y culturales. Para ello, Edge, Mendoza y Molina (2005) exponen que en la realización de las giras de campo se deben contemplar una serie de aspectos: propósito, objetivos, logística, inducción, mediación, participantes, discusión y acciones por seguir. Según López (s.f.), dichos aspectos no han sido contemplados formalmente en el diseño de las giras porque no se les ha dado una fundamentación didáctica interrelacionada con otras acciones antes, durante y después de la experiencia.

Las Giras de Campo como Estrategias de Enseñanza y Aprendizaje en las Ciencias Naturales. El campo de la educación en el manejo de los recursos naturales alberga, en cualquiera de sus espacios, un camino para la transformación de la sociedad, desde el desarrollo de una cultura que promueva el compromiso ante la problemática ambiental actual (Nieto, 2001). En este sentido, Tovar (2008) plantea la necesidad de iniciativas desde la dinámica de las ciencias naturales para proponer el aprendizaje, la enseñanza y la evaluación de la temática ambiental, por medio de la búsqueda de dimensiones conceptuales, metodológicas, actitudinales y comunicativas que respondan a los contextos políticos, ambientales, económicos y culturales cambiantes de las regiones y sus países.

Varios investigadores como Almeida (2008) y Protheroe y Clarke (2008) han diseñado estrategias de aprendizaje innovadoras y de instrucción que ayudan en los procesos de educación, pues mejoran el rendimiento de los estudiantes. Por esto, los autores resaltan la importancia de que los alumnos reconozcan este tipo de estrategias como herramientas para integrar la teoría a las prácticas en las que desarrollan sus habilidades.

Las actividades como las giras de campo posibilitan el aprendizaje significativo en áreas relacionadas con la protección y el manejo de los recursos naturales, como es el caso de la geología. Según López (s.f.) este tipo de estrategias es fundamental porque en ella se logra la interpretación científica de los principales fenómenos naturales, así como la aplicación del conocimiento adquirido. De hecho, es por medio del intercambio de experiencias de las giras de campo como se logra la interrelación de hechos, procesos y variables, y como se fomentan las actitudes y conductas positivas con el entorno y la conservación del ambiente.

Las giras de campo también son conocidas como excursiones o salidas de campo. Éstas son consideradas por Edge, et al. (2005) como un proceso de observación fuera de las horas habituales de la clase en el aula y que son realizadas con un propósito educacional en el que se proporciona a los alumnos una experiencia directa en un tema. Para su realización, Edge et al. (2005) recomiendan considerar una serie de aspectos, tales como: definir el propósito, hacer los arreglos necesarios, preparar la excursión con una mediación adecuada, procurar la participación de un conocedor del tema en la marcha de la visita y planear una discusión final que esté acompañada de acciones por seguir.

Ahora bien, desde el trabajo de la extensión rural, una gira de campo es entendida como una metodología para conocer situaciones reales de las condiciones productivas, con el fin de explorar posibles soluciones a los principales problemas encontrados. Ejemplos de giras de campo desde esta perspectiva son la visita a una finca, el día de campo, la gira educativa, una reunión o bien, demostraciones de métodos y resultados.

Por otra parte, López, Arévalo, Rodríguez, Andrade-Piedra y Quirós (2008) reconocen en las giras de campo la combinación de elementos teórico-prácticos y la participación de los actores 
directamente involucrados en las experiencias o visitas. Ellos indican que en éstas se genera el intercambio de conocimientos, el análisis, la reflexión y la evaluación. Así, definen una gira de campo como:

Una herramienta que facilita el conocimiento vivencial a través de encuentros con experiencias, sobre las que interesa entender su desarrollo, resultados y principales lecciones aprendidas, con el propósito de promover el intercambio horizontal de conocimientos entre distintos actores en un área específica y motivar el interés para la réplica de experiencias similares (p. 1).

En el trabajo de desarrollo comunitario participativo realizado por la FAO (2008), las visitas de campo o giras tecnológicas se entienden como el:

recorrido realizado a sistemas de producción agropecuarias, sitios de ecoturismo, plantaciones forestales o frutícolas, instalaciones agroindustriales, centros educativos, parcelas experimentales, granjas de diversos tipos, etc., que realiza un grupo determinado con el propósito de despertar el interés y actitudes progresistas enfocado a la solución de problemas que presentan en sus comunidades de origen (FAO, 2008, p. 2).

Este tipo de actividades busca que los participantes obtengan los aprendizajes necesarios en la resolución de presentes y futuros problemas. Dentro de sus objetivos de aprendizaje se encuentra el cambio de actitud, las habilidades técnicas en el manejo de alguna especie, la identificación de procesos, entre otros.

Para López (s.f.) las salidas o giras de campo son mucho más que un simple paseo o excursión: él reconoce las ventajas didácticas de este tipo de estrategias al mencionar que éstas pueden ser sumamente motivadoras para los estudiantes, pues les facilita la adquisición de habilidades, la formación científica y la educación ambiental, dentro de las ciencias naturales. Sin embargo, el mismo autor explica que este tipo de actividades generalmente no son integradas al currículo, razón por la cual no están planificadas y carecen de una fundamentación didáctica que esté interrelacionada con otras acciones antes, durante y después de la experiencia, como complemento al conocimiento del alumno en su proceso de enseñanza y aprendizaje.

Dentro de las consideraciones por tomar para el provecho académico de una gira de campo, López (s.f.) indica la importancia de que el alumno conozca cada una de las fases de la actividad, por lo que recomienda la explicación del contenido antes o al inicio de la gira, la descripción del lugar, la distancia, las generalidades logísticas, los materiales necesarios, las tareas por realizar, los protocolos científicos y los objetivos que se pretenden describir. De esta forma, al aclarar una serie de aspectos previos, los estudiantes pueden ejercer con destreza las habilidades requeridas, así como fomentar los periodos de reflexión, lo cual va a incentivar al alumno a exponer sus ideas y ejercer sus capacidades.

\section{Resultados}

Las generalidades obtenidas sobre el tema de las giras de campo se integraron como resultados en un marco de referencia para la determinación de las características técnico-pedagógicas tomadas en cuenta en el proceso del material multimedial, así como de los posibles usos académicos esperados. Las principales opiniones de los participantes fueron resumidas y redactadas en apego a los comentarios de los participantes.

Sobre el concepto de una gira de campo. Primeramente, se conversó sobre las generalidades de las giras de campo, su propósito académico y cómo podrían clasificarse en la institución de estudio. En resumen, y de acuerdo con las opiniones expresadas por los informantes, las giras de campo se definieron como actividades complementarias a las tutorías y materiales didácticos, en las cuales se fortalecen los conocimientos teóricos de los estudiantes. Asimismo, se definieron como espacios en los que se logra la implementación de aspectos transversales que integran la docencia, la investigación y la extensión, por medio de encuentros presenciales, en los que se genera la interrelación entre 
profesores, estudiantes y los actores sociales de la comunidad.

Estos aspectos mencionados exponen a los estudiantes a la realidad de procesos y situaciones que, como profesionales, tendrán que enfrentar en su futuro campo de acción; de manera que el propósito académico de una gira de campo es, entonces, exponer a los estudiantes a una situación práctica para valorar, evaluar y analizar el nivel de dominio de una capacidad y habilidad, más allá de los aspectos teóricos aprendidos y teniendo en cuenta los diferentes estilos de aprendizaje de cada alumno.

Los participantes indicaron que en la institución en estudio (con un modelo de educación a distancia), se podrían categorizar las giras de campo de acuerdo a los siguientes criterios:

a. Por la forma de realización: sea obligatoria, en grupos, bajo la guía de un profesor asignado o bien, bajo un formato de autogira, siguiendo los parámetros predeterminados en el curso.

b. Por su propósito: 1) académica, tales como de observación, monitoreo, recolección, práctica; 2) administrativa, considerada para la prospección de los docentes; 3) investigativa, para gestión y generación de conocimiento y 4) de extensión de proyectos, programas de ayuda y capacitación de actores sociales.

Otras de las menciones realizadas por los informantes para una posible clasificación de las giras de campo fue: a) el destino y sitio de visita; b) la época estacional (para observar el mismo paisaje, pero desde la interacción de condiciones climáticas diferentes); c) la duración (horas, días) y d) la dificultad o riesgo, así como por las habilidades requeridas por los estudiantes.

La evaluación en las giras de campo. Los informantes expusieron que predominantemente se aplica una evaluación sumativa al final de la actividad. La parte formativa es realizada durante la gira de campo y tiene el propósito de medir el grado de aprendizaje y la actitud demostrada por el estudiante en su trabajo individual o en equipo.

Los encargados de cátedra y profesores expresaron que generalmente se le solicita al estudiante la entrega de un reporte como informe final. Por su parte, algunos profesores indicaron realizar conversatorios al final de la gira, ya sea presenciales o por medio de foros en las plataformas de aprendizaje de la institución, para que los estudiantes compartan sus experiencias, aprendizajes, fotos, imágenes, comentarios y anécdotas.

Algunos de los participantes rescataron la importancia de aprovechar los informes y resultados de los estudiantes en las giras de campo realizadas, porque consideran que es el espacio en el que hay una realimentación y se genera la verdadera reflexión de la actividad o el curso.

Papel de las giras de campo dentro del diseño curricular del curso. $\mathrm{Al}$ respecto, el encargado de programa indicó que todos los cursos de la carrera cuentan con su respectivo diseño curricular. Además, los asesores curriculares y encargados de cátedra indicaron las principales partes que compone el diseño curricular de la institución en estudio: a) una presentación general con la información del curso (nombre, código, naturaleza del curso, ejes temáticos y transversales, créditos, horas, responsables, propósito del curso, ubicación en el plan de estudios, recursos didácticos, metodología, distribución de horas, orientaciones didácticas, evaluación, gestión administrativa, referencias bibliográficas); b) una plantilla en donde se distribuyen en columnas distintas los objetivos de aprendizaje, la descripción de los contenidos, las estrategias metodológicas y actividades de aprendizaje, la relación de medios y recursos didácticos y la evaluación y autorregulación de los aprendizajes. En esta segunda plantilla se da la oportunidad de indicar o especificar las actividades a realizar, tales como la gira de campo.

Los participantes coincidieron en que en el diseño curricular no se puede explicar la realidad que se vive en la práctica de una gira de campo, ya que este presenta una estructuración estándar de las giras de campo como actividades de aprendizaje, por lo cual mencionaron la 
importancia de incorporar diseños didácticos o instruccionales a las actividades de aprendizaje como las giras de campo.

Siguiendo el apartado anterior, los participantes recordaron y explicaron algunas de las indicaciones que generalmente les dan a los estudiantes para realizar las giras de campo, las cuales son tratadas en las orientaciones de los cursos. En dichas indicaciones se abordan temas como los objetivos, los requerimientos, los porcentajes de evaluación, entre otros; sin embargo, como ya se dijo, sí existe la necesidad de contar con materiales instruccionales previos a las giras de campo, tanto para profesores como para los estudiantes.

Los participantes coinciden en la falta de materiales didácticos o instruccionales que guíen la labor del profesor en las giras de campo. Entre los aspectos señalados se mencionaron los siguientes: a) señalamiento de planes alternativos en caso de alguna eventualidad en la realización de las giras; b) especificaciones de los lugares a visitar; c) cantidad máxima de estudiantes por gira, d) técnicas de formación de grupos; asignación y tareas de los asistentes de las giras; e) logística de transporte, alimentación y hospedaje; f) evaluaciones sugeridas; g) manejo y planeación de cronograma de actividades con el señalamiento de responsables de cada fase de las giras de campo.

Asimismo, los informantes manifestaron la necesidad de materiales instruccionales que guíen al estudiante con indicaciones de observación, rúbricas de evaluación, guía para la presentación del informe, reglamentos, normativa, protocolo de comportamiento, aspectos de seguridad a contemplar, equipo requerido, materiales, orientaciones, tipo de ropa a llevar, advertencia de seguridad (normativa internacional y médica); metodología en la entrega de informe, requisitos y pre-requisitos, aspectos de primeros auxilios, consideraciones de salud, problemas psicológicos, explicación de las etapas a realizar, entre otros.

Fases o etapas en que se puede dividir una gira de campo. En el mismo orden de temas, los participantes indicaron, a través de una construcción colectiva oral, que las giras de campo se pueden dividir en cuatro fases o etapas:

a. Fase inicial: se den instrucciones, material impreso, conocimientos previos, objetivos, lineamientos generales, guía, entre otros.

b. Fase de ejecución: se genera la práctica, para lo cual se debe contar con una guía de seguimiento, rúbrica e instrumentos que permitan describir lo observado y lo medido.

c. Fase de recopilación de la información o elaboración del informe.

d. Fase de socialización de la experiencia: se comparte la experiencia. Puede ser por medio de los recursos tecnológicos disponibles en la institución como las plataformas de aprendizaje. Para ello, se recurre a foros, blogs o bien, presencialmente en una discusión o realimentación en grupo (tutoría).

Materiales didácticos para las giras de campo. Los informantes señalaron la existencia y disponibilidad de materiales didácticos en video para algunos de los cursos con giras de campo del programa de estudio (montañismo e incendios forestales); no obstante, tanto el encargado de programa como los encargados de cátedra y algunos profesores coincidieron en la importancia de desarrollar materiales multimediales instruccionales en los que se presenten técnicas, procedimientos, recomendaciones y explicaciones; se prepare a los estudiantes para las experiencias que se desarrollarán, y se abarquen temas como: a) concepto de una gira de campo, b) objetivo de la gira de campo, c) explicación de los pasos o fases, d) asuntos de seguridad, e) qué cosas se deben hacer y qué no, f) qué ropa se debe llevar, f) cuál es el equipo básico, f) qué tipo de equipo, h) aspectos generales del lugar a visitar, i) condiciones del lugar a visitar, h) guía de trabajo; i) protocolos y reglamentos a seguir por todos los participantes, $\mathrm{j}$ ) varia información como mapa, horario, cronograma, fuentes de información importantes de estudiar, sitios web que visitar.

Metodologías y didácticas. Los participantes recomendaron implementar metodologías 
adecuadas mediante las tendencias del trabajo colaborativo, de apoyo o de comparación, en especial para la opción de autogira. Se señalan aspectos que contribuyen a la calidad de la realización de las giras de campo, como: a) la atención personalizada, b) la planificación, c) las instrucciones claras y precisas y d) el lenguaje técnico, pero apropiado para los estudiantes.

\section{Discusión}

Desde una de las nuevas relaciones de la enseñanza de las ciencias naturales, se perciben las giras de campo como escenarios académicos. Las giras de campo adquirieron un reconocido valor en el estudio, al tomarse como parte importante de la formación de los futuros profesionales en el manejo de los recursos naturales, razón por la cual se valoró cómo estas deberían incorporarse a un modelo de educación a distancia.

Así pues, las giras de campo se consideraron como escenarios académicos para generar una vivencia de aprendizaje, en la cual se promueva que los estudiantes se empoderen del conocimiento teórico y lo puedan poner en práctica bajo condiciones reales. Lo anterior corrobora lo planteado por López et al. (2008), quienes señalan la fortaleza del intercambio de conocimiento, el análisis, la reflexión y la evaluación de las giras de campo. Ellos las definen de la siguiente manera:

Una herramienta que facilita el conocimiento vivencial a través de encuentros con experiencias, sobre las que interesa entender su desarrollo, resultados y principales lecciones aprendidas, con el propósito de promover el intercambio horizontal de conocimientos entre distintos actores en un área específica y motivar el interés para la réplica de experiencias similares (p. 1).

Asimismo en un escenario más cercano a Costa Rica, Córdoba (2008) explica la importancia de las excursiones educativas en Panamá "como estrategia metodológica de aprendizaje, no solo en la enseñanza de la Historia, sino también de otras disciplinas académicas, en vista a los resultados obtenidos en las experiencias expuestas" (p. 3692).
Los resultados indicaron que las giras de campo pueden ser clasificadas según varios criterios y parámetros. Los informantes brindaron un marco para un primer ordenamiento relacionado con el propósito buscado dentro de tres grandes áreas: docencia, investigación y extensión de la carrera en estudio en la sociedad costarricense. Para el caso de actividades que se realizan en la institución, desde la docencia fueron indicadas otras posibles sub-clasificaciones, sea por ejemplo bajo criterios como duración de la actividad, riesgo inherente, localización geográfica, costos, entre otras.

En la institución de estudio no se encontraron documentos oficiales en donde se exponga una clasificación para las giras de campo; por lo que la propuesta de realizar este esfuerzo de ordenamiento de categorías será una importante innovación, específicamente para la carrera de MARENA. Al contar con tal clasificación, se podrán facilitar criterios de decisión en la gestión académica de este tipo de actividades; por ejemplo la selección de la calendarización de las giras (sobre todo en un país en donde imperan dos estaciones tropicales de verano e invierno). También, se contará con parámetros para medir costo y objetivos, cantidad de estudiantes, cantidad de profesores y asistentes, requerimientos y facilidad de equipo, previsiones de seguridad, entre otras ya mencionadas en el capítulo de resultados.

En el planeamiento de las giras de campo, no sólo es necesaria una clasificación de las mismas, sino que también es importante definir las etapas básicas en su realización. En la investigación realizada se pudo observar una relación de las etapas con el uso de los enfoques de las teorías de aprendizaje.

Generalmente, las giras de campo en la carrera de estudio son desarrolladas en cuatro fases o etapas. En la primera etapa, los profesores dan indicaciones, instrucciones e información teórica (de enfoque predominantemente conductista). En la segunda, se ejecuta o desarrolla la gira de campo (generalmente bajo una aplicación enfocada desde la cognición situada). Por último, la tercera y cuarta son caracterizadas por la recopilación de la información y la socialización de 
la experiencia (de connotación dentro de los enfoques cognitivo crítico social y constructivista).

En forma de analogía, las etapas descritas pueden corresponder a los pasos en que los estudiantes realizarían el acercamiento al objeto de estudio, a través de una práctica evolutiva de los enfoques de las teorías de aprendizaje en el estudiante, llamadas por Driscoll (2005) como conductista, cognitivista y constructivista. En las actividades vivenciales se generaría así la construcción de un conocimiento gradual, aprovechando la observación, la experimentación y la aplicación e integrando, de esa forma, motivadores internos y externos en el aprendizaje del estudiante Méndez (2006).

Fue precisamente los aspectos señalados de falta de clasificación y definición de etapas de las giras de campo, lo que permitió visualizar la falta de una fase de diseño instruccional como solución para la planificación de este tipo de actividades.

En este sentido, los resultados permiten visualizar la necesidad de tomar decisiones respecto de los procesos existentes para el currículo dentro de la universidad en estudio, como lo son los diseños instruccionales de los cursos del programa de estudio.

En tal institución, se implementa un formato de diseño curricular en el cual se detallan aspectos generales de la asignatura a ofertarse, y dentro de los cuales se destina un espacio vertebral (columnar) para la mención de estrategias de aprendizaje. Sin embargo, no es un diseño instruccional. Al respecto, los informantes recalcaron la imposibilidad de integrar en el diseño curricular actual especificaciones de las actividades de aprendizaje de los cursos, las cuales deberían reflejar la complejidad de la realización de actividades presenciales vivenciales, como lo son las giras de campo.

En este sentido, sin un diseño instruccional en los cursos con giras de campo, es evidente que la planeación y realización de este tipo de actividades responden parcialmente a las necesidades de aprendizaje y a los objetivos planteados en el diseño curricular. De allí la serie de recomendaciones brindadas por los informantes como parte de sus posibles mejoras. De igual manera, al no existir un diseño instruccional que oriente a docentes y estudiantes, se deja al azar una serie de aspectos que ponen en riesgo el aprovechamiento del potencial de una experiencia vivencial, así como de los resultados en la formación del perfil de salida de los estudiantes. Es meritorio recordar que las teorías instruccionales para Guerrero y Flores (2009) se enfocan en la práctica, pues esto le permite al diseñador o docente contar con una visión clara de "la forma en que se pueden lograr los objetivos que se plantean" (p. 319).

Aunado a lo anterior, las orientaciones del curso, documento que se entrega a los estudiantes una vez que lo matriculan, no fungen como una manual instruccional para el docente.

Como puede verse, entonces, el proceso faltante en la universidad en estudio es el diseño instruccional, por su especificidad en la planificación de un curso. El establecimiento de una fase en la que se contemple este diseño puede mejorar el planteamiento del desarrollo de los cursos y sus diferentes actividades de aprendizaje; así como ofrecer guías y directrices para los docentes en cuanto a la necesidad de producción de materiales didácticos.

Sin el aporte del diseño instruccional en los cursos y la debida planeación de las actividades de aprendizaje vivenciales, se comete el error de recargar las responsabilidades en el docente, quien más bien en una actividad vivencial debería optar por un rol de facilitador y observador. En este sentido, López (s.f.) indica que con una debida instrucción de las tareas a realizar, se evita la excesiva participación y actuación del profesor en la experiencia de la actividad presencial.

Ahora bien, además de las razones expuestas, se lograron identificar situaciones que respaldan la necesidad de diseño instruccional. En primer lugar, la poca utilización de estrategias de acompañamiento al estudiante con medios y formatos alternativos al documento impreso y a la tutoría presencial. En segundo lugar, el uso predominante de una evaluación sumativa en las giras de campo, la cual no deja espacio para la evaluación formativa, actitudinal, de reflexión y realimentación para los estudiantes. En tercer lugar, la necesidad de aspectos de mejora general en la realización de las giras de campo 
(necesidad de planes alternativos a situaciones inesperadas (plan A, B o C); especificaciones de los lugares a escoger para la realización de las giras; definición de la cantidad máxima de estudiantes por gira; asignación de asistentes en las giras; mejora de la logística de transporte, alimentación y hospedaje; evaluación sugerida; cronogramas de actividades detallados; especificación de encargados y responsables de las actividades a desarrollar; establecimiento de una periodicidad recomendada para cada gira de campo por oferta de curso; planteamiento de giras colegiadas (verificar que las fechas de las giras no choquen entre sí); reglamento, normativa y protocolo; así como aspectos de seguridad).

Los detalles de falta de planeación en los cursos con giras de campo en aspectos de seguridad, dejó planteada la necesidad de políticas, normativa y regulación en la gestión administrativa y académica que respalda la realización de las giras de campo. En la investigación no se encontraron mayores evidencias de la existencia de políticas, nomenclaturas o regulaciones referentes a la realización de las giras de campo en la universidad de estudio. En este sentido, Simonson (2009) considera que las políticas son el curso de acción que facilita el desarrollo de un programa de estudio en un modelo de educación a distancia. Estas son los parámetros que establecen el acuerdo entre las partes y actores que interactúan en una institución académica; de allí la marcada necesidad de regulaciones que muestren los roles, derechos y responsabilidades de los actores -sean estudiantes, profesores, personal administrativo y académico, en las actividades vivenciales tales como las giras de campo.

\section{Conclusiones y Recomendaciones}

Como ya se expresó anteriormente, un modelo universitario de educación a distancia no excluye actividades presenciales como laboratorios, prácticas y giras de campo. En dichas actividades se generan situaciones de riesgo dentro y fuera de las instalaciones institucionales; por ejemplo, el traslado de personas o la permanencia fuera del campus por horas o días, en las cuales las personas están expuestas a cualquier tipo de accidentes físicos, que tarde o temprano pueden acarrear problemas de tipo legal a la institución. En este sentido, es necesaria la aprobación de políticas, normativas y regulaciones en la administración educativa, con el propósito de prevenir problemas antes de que se dé la actividad real, tal y como lo mencionan Robbins y Coulter (2011).

Se recomienda que las giras de campo sean planificadas y organizadas pedagógicamente, como estrategias de aprendizaje desde el diseño curricular y, por ende, que cuenten con un diseño instruccional particular.

Es de vital importancia la recapitulación de conceptos, definiciones, enfoques y características; así como la clasificación de las giras de campo para la formulación de manuales de ayuda al docente y al estudiante, dado que cada una de las giras cumple diferentes propósitos para cada curso y para cada gira de campo en sí. De esta forma, se puede promover una clasificación que provea información sobre sus características (duración, desplazamiento, habilidades requeridas o riesgos implícitos en su realización), para lo cual se hace necesaria la elaboración de políticas y reglamentos que amparen la realización de actividades vivenciales en un modelo de educación a distancia.

Los materiales multimediales deben ser una herramienta que facilite el papel del docente a cargo de giras de campo, de tal manera que no esté sobrecargado con la exposición de temas, especificaciones o explicaciones que pueden realizarse por otro tipo de medios tecnológicos.

En cuanto a estrategias de evaluación, se recomienda el análisis de la evaluación horizontal para giras de campo, propuesta por la Alianza Cambio Andino (2008), de acuerdo con la cual se considera la evaluación horizontal como "un método flexible de evaluación que combina la autoevaluación y una revisión externa por "pares". La participación de "pares" neutraliza las relaciones de poder asimétricas que prevalecen en evaluaciones externas tradicionales, creándose una atmósfera más favorable para aprender y mejorar" (p. 2).

Asimismo, se exhorta a la aplicación de otras estrategias pedagógicas en la planificación 
del diseño instruccional de las giras de campo; por ejemplo, la inclusión de trabajos colaborativos, de apoyo y de comparación, con el uso de los recursos de las TIC institucionales.

En la determinación de las etapas de las giras de campo por parte de los informantes (tales como etapa inicial, de ejecución y/o desarrollo, recopilación de información y socialización de experiencia), se pueden alternar las sugerencias metodológicas y didácticas de la Alianza Andina (2008); las cuales pueden ser aplicadas dependiendo del tipo de gira de campo a desarrollar. De acuerdo con las etapas y alternativas didácticas en la realización de las giras de campo frente a una opción de sistematización de experiencias, la Alianza Cambio Andino (2008) establece lo indicado a continuación:

a. En la presentación conceptual y teórica sobre las experiencias a desarrollar en la visita, se puede utilizar alternativamente un panel de expertos.

b. En la visita a experiencias con protagonistas, entrevistas semiestructuradas o espacios orientados a comentarios y preguntas, se pueden preparar reuniones con organizaciones vinculadas con un rol en el desarrollo o institucionalización de la experiencia, con el fin de hacer entrevistas por grupos y compartir resultados al final de la jornada.

c. En la visita o reunión número dos y en el espacio orientado a preguntas y comentarios, se puede realizar una visita para conocer el contexto temático o bien, visitas complementarias a lugares o actores mencionados en la reunión.

d. En el cierre de la jornada, se puede desarrollar un taller para identificar y sistematizar nuevos aprendizajes (diario) y para presentar conclusiones, así como la evaluación final de la gira.

Se aconseja al programa en estudios generar acciones con el propósito de contar con una respuesta a las peticiones de los informantes con respecto a:
- Planes alternativos a situaciones inesperadas (plan A, B o C); b) especificaciones de los lugares a escoger para la realización de las giras; c) pronunciamientos sobre cantidad máxima de estudiantes por gira; d) asignación de asistentes en las giras; e) especificaciones y regulación sobre la logística de transporte, alimentación y hospedaje de las giras de campo; f) estandarización colegiada sobre la evaluación sugerida; g) especificaciones de cronogramas de actividades detallados para cada curso y gira de campo; h) especificaciones y regulación sobre los encargados y responsables de las actividades a desarrollar; i) indicaciones de la periodicidad recomendada para cada gira de campo por oferta de curso; j) planteamiento de giras colegiadas (verificar que las fechas de las giras no choquen entre sí); k) reglamento, normativa, protocolo en las giras de campo; 1) aspectos de seguridad a contemplar, y m) reglamento de las giras de campo.

\section{Referencias}

Alianza Cambio Andino (2008). Giras de Aprendizaje. Recuperado de http://www.cambioandino.org/girasdeaprendizaje.shtml

Almeida, L. (2008). The effects of different learning strategies to facilitate achievement of different educational objectives. [Los efectos de diferentes estrategias de aprendizaje para facilitar el logro de diferentes objetivos educativos]. TechTrends: Linking Research \& Practice to Improve Learning. 52(3), 32-36. doi:10.1007/s11528-008-0152-y

Córdoba, A. (2008). La importancia de las excursiones educativas como estrategia didáctica en la enseñanza de la historia. En IX Congreso Centroamericano de Historia. San José, Costa Rica: Universidad de Costa Rica. Recuperado de http://www.historia.fcs.ucr.ac.cr/ articulos/2008/especial2008/articulos/12-Teoria/150. pdf

Creswell J.C. (2003). Research Design, qualitative, quantitative, and mixed methods approaches [Diseño de investigación, cualitativo, cuantitativo y desarrollo de métodos mixtos]. (2da ed.). E.E.U.U.: Sage Publications. 
Creswell, J., \& Plano, V. (2007). Designing and conducting mixed methods research.[Diseñando y conduciendo el diseño de investigaciones de métodos mixtos] (2da ed.). EE.UU.: Sage Publications.

Driscoll, M. (2005). Psychology of learning for instruction. [Psicología del aprendizaje de la instrucción] (3ra ed.). EE.UU.:Pearson Education.

Edge, F., Mendoza, E., \& Molina, S. (2005). Metodología pedagógica: Once técnicas de enseñanza que todo educador debe conocer y practicar. Colombia: Casa Bautista de Publicaciones.

Food and Agriculture Organization [Organización de las Naciones Unidas para la Agricultura y la Alimentación]. (Enero, 2008). Gira de campo FAOMAGA Sistematizaciones FAO. Representación Guatemala FAO 3, 1-9. Recuperado de http://www. rlc.fao.org/iniciativa/pdf/faoguat08.pdf y de http:// www.rlc.fao.org/es/prensa/boletines/pdf/4.pdf

Gómez, M. (2006). Introducción a la metodología de la investigación científica. Argentina: Editorial Brujas.

Guadamuz, L. (2008). Prólogo. En Lobo, N., \& Fallas, V.U. La Benemérita Universidad Estatal a Distancia en la sociedad del conocimiento, (pp. XXV-XLV). San José, Costa Rica: EUNED.

Guerrero, T., \& Flores, H. (abril, mayo, junio, 2009). Teorías de aprendizaje en el diseño de materiales didácticos informáticos. Artículos Arbitrados EDUCERE, Año 13(45), 317-329.

Hernández, R., Fernández, C., \& Baptista, P. (2006). Metodología de la Investigación. (4ta ed.). México: McGraw Hill Interamericana.

Kirshner, D., \& Whitson, J.A. (Eds.), (1997). Editor's introduction. Situated cognition: Social, semiotic, and psychological perspectives. [Cognición situada: Social, semiótica y perceptiva psicológica]. EE.UU.:Erlbaum.

López, G., Arévalo, D., Rodríguez, F., AndradePiedra, J., \& Quirós, C. (2008). Gira de aprendizaje: Enfoque participativo de cadenas productivas. EPCP, 1, 1-4. Recuperado de http:// www.cambioandino.net/aca/c/document_library/get_ file ?folderId $=85 \&$ name $=$ DLFE-45. doc -
López, J.A., (s.f.). La salida de campo: mucho más que una excursión. Recuperado de www.educarm.es/templates/portal/.../11/22_salidasdecampo.pdf

Méndez, V.H., \& Monge, J. (2006). La enseñanza de los modelos ecológicos de depredación a través de un laboratorio virtual. XII Congreso Internacional de Tecnología y Educación a Distancia. San José, Costa Rica: UNED. Recuperado de http://www.uned.ac.cr/ globalNet/global/tecnologia/transmision/articulos/ensenanzadelosmodelos.htm

Naghi, M. (2005). Metodología de la Investigación. (2da ed.). México: Limusa.

Nieto, L.M. (2001). Modalidades de la educación ambiental: diversidad y desafíos. En dos Santos, J.E. \& Michéle S. A Contribuicao da Educao Ambiental a Esperanca de Pandora. Brasil: Rima Editora. Recuperado de http://ambiental.uaslp.mx/docs/ LMNC-AI-01-ModEALibro.pdf

Protheroe, N. \& Clarke, S. (2008). Learning strategies as a key to student success. [Estrategias de aprendizaje como clave para el éxito de los estudiantes]. Principal, 88(2), 33-37. Recuperado de Education Research Complete. http://www.eric. ed.gov/ERICWebPortal/custom/portlets/recordDetails/detailmini.jsp?_nfpb=true\&ERICEstSearch SearchValue_0=EJ $823093 \&$ ERICExtSear ch_SearchType_0=no\&accno=EJ823093

Robbins, S.,\& Coulter, M. (2011). Administración (3era. Ed.). México: Pearson Educación.

Simmonson, M. (2009). Institutional policy issues for distance education. [Retos de las políticas de la educación a distancia]. NOVA. EE.UU. Recuperado de http://www.schoolofed.nova.edu/bpol/pdf/policy_issues.pdf

Tashakkori, A., \& Teddlie, Ch. (1998). Mixed methodology: Combining qualitative and quantitative approaches. [Metodología mixta. Combinando estudios cualitativos y cuantitativos]. EE.UU.: Sage Publications.

Tovar, J.C. (5 de setiembre, 2008). Propuesta de modelo de evaluación multidemensional de los aprendizajes en ciencias naturales y su relación con la estructura de la didáctica de las ciencias. Revista Eureka sobre Enseñanza y Divulgación de las Ciencias. 003, 259273. Asociación de Profesores Amigos de la Ciencia. 
Cádiz, España. Eureka. En Red de Revistas Científicas de América Latina y el Caribe, España y Portugal. Universidad Autónoma del Estado de México. Redalyc. Recuperado de http://redalyc.uaemex.mx/ redalyc/src/inicio/ArtPdfRed.jsp?iCve=92050302

Universidad Estatal a Distancia. (2008a.). Información General 2009. San José, Costa Rica: EUNED
Universidad Estatal a Distancia. (2008b.). Informe de Autoevaluación: Presentado ante el Sistema Nacional de Acreditación de la Educción Superior de Costa Rica, SINAES, para optar por la Acreditación de la carrera: Bachillerato y Licenciatura en Protección y Manejo de Recursos Naturales. Escuela de Ciencias Exactas y Naturales. 1-132. Vicerrectoría Académica. San José, Costa Rica: UNED. 